\title{
Asymptotic study of a frictionless contact problem between two elastic bodies
}

\author{
Y. Letoufa ${ }^{a}, H$. Benseridi $^{b, *}$, M. Dilmi ${ }^{b}$ \\ ${ }^{a}$ Applied Mathematics Laboratory, El Oued University, 39000, Algeria. \\ ${ }^{b}$ Applied Mathematics Laboratory, Setif 1 University, 19000, Algeria.
}

\begin{abstract}
We consider a mathematical model which describes the bilateral, frictionless contact between two elastic bodies. We will establish a variational formulation for the problem and prove the existence and uniqueness of the weak solution. We then study the asymptotic behavior when one dimension of the domain tends to zero. In which case, the uniqueness result of the solution for the limit problem are also proved. (C)2016 All rights reserved.
\end{abstract}

Keywords: A priori inequalities, free boundary problems, nonlinear equation, transmission conditions, Tresca law, variational problem.

2010 MSC: 35R35, 76F10, 78M35.

\section{Introduction}

In this paper, we study a problem involving boundary conditions describing real phenomena such as contact and friction between two elastic bodies. The problem presented in this work is very frequent in applications. For instance the physical domains are defined such that the height is much smaller than the length. These are the assumptions of elasticity and Visco-elasticity of a tire. The model for the lassa hemorrhagic fever and the model of the groundwater owing within a leaky aquifer [1, 2]. Other applications are related to the mechanism of ball bearing. Scientific researches in mechanics are articulated around two main components: one devoted to the laws of behavior

\footnotetext{
* Corresponding author

Email addresses: letoufa54@gmail.com (Y. Letoufa), m_benseridi@yahoo.fr (H. Benseridi), mouraddil@yahoo.fr (M. Dilmi)
} 
and the other on boundary conditions imposed on the body. Several works have been done on the mechanical contact with the various laws of behavior and various friction boundary conditions close to our problem, however these papers were restricted only to the results of existence and uniqueness of the weak solution under several assumptions. Let us mention for example the work by [12] in which the authors obtained the existence and uniqueness result by construction of an appropriate mapping which is shown to be a contraction on a Hilbert space. Other similar problems can be found in monographs such as [13, and the literature quoted there. In the last few years, some research papers have been written dealing with both the asymptotic analysis of an incompressible fluid in a three-dimensional thin domain, when one dimension of the fluid domain tends to zero can be found in [7-10]. The authors in [5] studied the asymptotic and numerical analysis for a unilateral contact problem with Coulomb's friction between an elastic body and a thin elastic soft layer. More recently, the asymptotic analysis of a dynamical problem of isothermal elasticity with non linear friction of Tresca type was studied in [6]. The asymptotic convergence of a dynamical problem of a non-isothermal linear elasticity with friction were studied in [16]. The numerical solutions of this type of problem are studied in e.g. [14, 15]. Furthermore, the authors in [3] have given a new hyperbolic function solutions for some nonlinear partial differential equation arising in mathematical physics. The goal of this paper is to study the asymptotic behavior of a boundary value problem in a three dimensional thin domain $\Omega^{\varepsilon}$ with non linear friction of Tresca type. The novelty here consist in the fact that we study the contact between two bodies $\left(\Omega^{\varepsilon}=\Omega_{1}^{\varepsilon} \cup \Omega_{2}^{\varepsilon}\right)$ and we assume that on the common part of the boundary there is no separation between the bodies during the process, that is, the contact is bilateral. The use of the small change of variable $z=\frac{x_{3}}{\varepsilon}$, transforms the initial problem posed in the domain $\Omega^{\varepsilon}$ into a new problem posed on a fixed domain $\Omega=\Omega_{1} \cup \Omega_{2}$ independent of the parameter $\varepsilon$. We prove some estimates on the displacement independent of the small parameter. The passage to the limit on $\varepsilon$, permits us to obtain the existence and uniqueness of the limit of a weak solution to the problem described in the abstract.

This article is organized as follows. In Section 2 we introduce some notations and preliminary. In Section 3 we describe the model and present its variational formulation. In Section 4 we find some estimates and prove convergence theorem by using several inequalities. Finally, we obtain all the properties of our original problem.

\section{Notations and preliminaries}

We denote by $S_{3}$ the space of second order symmetric tensors on $\mathbb{R}^{3}$ and |.| the Euclidean norm on $\mathbb{R}^{3}$. Thus, for every $u, v \in \mathbb{R}^{3}, u \cdot v=u_{i} v_{i},|v|=(v \cdot v)^{1 / 2}$, and for every $\sigma, \tau \in S_{3}, \sigma \cdot \tau=\sigma_{i j} \tau_{i j}$, $|\tau|=(\tau, \tau)^{1 / 2}$. Here and below, the indices $i$ and $j$ run between 1 and 3 and the summation convention over repeated indices is adopted.

Let $\Omega_{1}^{\varepsilon}$ and $\Omega_{2}^{\varepsilon}$ be two bounded domains in $\mathbb{R}^{3}$. Everywhere in this paper, we use a superscript $l$ to indicate that a quantity is related to the domain $\Omega_{l}^{\varepsilon}, l=1,2$, where $(0<\varepsilon<1)$ is a small parameter that will tend to zero. For each domain $\Omega_{l}^{\varepsilon}$, we assume that its boundary $\partial \Omega_{l}^{\varepsilon}$ is the class $C^{1}$ and is partitioned into three disjoint measurable parts, which $\partial \Omega_{1}^{\varepsilon}=\bar{\omega} \cup \bar{\Gamma}_{1}^{\varepsilon} \cup \bar{\Gamma}_{L_{1}}^{\varepsilon}$ and $\partial \Omega_{2}^{\varepsilon}=\bar{\omega} \cup \bar{\Gamma}_{2}^{\varepsilon} \cup \bar{\Gamma}_{L_{2}}^{\varepsilon}$, where $\omega$ is fixed region in the plane $x^{\prime}=\left(x_{1}, x_{2}\right) \in \mathbb{R}^{2}$. The upper surface $\Gamma_{1}^{\varepsilon}$ is defined by $x_{3}=\varepsilon h\left(x^{\prime}\right)$, and the upper surface $\Gamma_{2}^{\varepsilon}$ is defined by $x_{3}=-\varepsilon h\left(x^{\prime}\right)$ where $h$ is a smooth bounded function such that $0<h_{*} \leq h\left(x^{\prime}\right) \leq h^{*}$ for all $\left(x^{\prime}, 0\right)$ in $\omega$. $\Gamma_{L_{l}}^{\varepsilon}, l=1,2$ is the lateral boundary. We denote by $\Omega^{\varepsilon}$ the domain $\Omega_{1}^{\varepsilon} \cup \Omega_{2}^{\varepsilon}$ and we put

$$
\begin{aligned}
& \Omega_{1}^{\varepsilon}=\left\{\left(x^{\prime}, x_{3}\right) \in \mathbb{R}^{3},\left(x^{\prime}, 0\right) \in \omega, \quad 0<x_{3}<\varepsilon h\left(x^{\prime}\right)\right\}, \\
& \Omega_{2}^{\varepsilon}=\left\{\left(x^{\prime}, x_{3}\right) \in \mathbb{R}^{3},\left(x^{\prime}, 0\right) \in \omega, \quad-\varepsilon h\left(x^{\prime}\right)<x_{3}<0\right\} .
\end{aligned}
$$


We shall use the notation

$$
\begin{aligned}
H^{1}\left(\Omega_{l}^{\varepsilon}\right)^{3} & =\left\{\mathbf{v} \in L^{2}\left(\Omega_{l}^{\varepsilon}\right)^{3}: \frac{\partial v_{i}}{\partial x_{j}} \in L^{2}\left(\Omega_{l}^{\varepsilon}\right), \forall i, j=1, \ldots, 3\right\}, \\
V\left(\Omega_{l}^{\varepsilon}\right) & =\left\{\mathbf{v} \in H^{1}\left(\Omega_{l}^{\varepsilon}\right)^{3}: \mathbf{v}=0 \text { on } \Gamma_{l}^{\varepsilon} \cup \Gamma_{L_{l}}^{\varepsilon}\right\}, l=1,2 .
\end{aligned}
$$

The spaces $H^{1}\left(\Omega_{l}^{\varepsilon}\right)^{3}$ and $V\left(\Omega_{l}^{\varepsilon}\right)$ are real Hilbert spaces. All these spaces are endowed with their natural norms $\|.\|_{1 . \Omega_{l}^{\varepsilon}}$ and scalar product $\langle., .\rangle_{1 . \Omega_{l}^{\varepsilon}}$.

Moreover, we need the following functional spaces:

$$
V^{\varepsilon}=\left\{\left(\mathbf{v}_{1}, \mathbf{v}_{2}\right) \in V\left(\Omega_{1}^{\varepsilon}\right) \times V\left(\Omega_{2}^{\varepsilon}\right): \mathbf{v}_{1} \cdot \nu_{1}+\mathbf{v}_{2} \cdot \nu_{2}=0 \text { on } \omega\right\} .
$$

The spaces $V^{\varepsilon}$ is real Hilbert spaces endowed with the canonical inner products $\langle., .\rangle_{V^{\varepsilon}}$ and the associated norms $\|(., .)\|_{V^{\varepsilon}}$, where

$$
\left\|\left(\mathbf{v}_{1}, \mathbf{v}_{2}\right)\right\|_{V^{\varepsilon}}=\left(\left\|\mathbf{v}_{1}\right\|_{V\left(\Omega_{1}^{\varepsilon}\right)}^{2}+\left\|\mathbf{v}_{2}\right\|_{V\left(\Omega_{2}^{\varepsilon}\right)}^{2}\right)^{1 / 2} .
$$

We still denote the norm of the space $H^{1}\left(\Omega_{1}^{\varepsilon}\right)^{3} \times H^{1}\left(\Omega_{2}^{\varepsilon}\right)^{3}$ by $\|(., .)\|_{1, \Omega_{1}^{\varepsilon} \times \Omega_{2}^{\varepsilon}}$. Since the boundary $\partial \Omega_{l}^{\varepsilon}$ is Lipschitz continuous, the unit outward normal vector $\nu_{l}$ on the boundary $\partial \Omega_{l}^{\varepsilon}$ is defined a.e.. For every vector field $\mathbf{v}_{l}^{\varepsilon} \in H^{1}\left(\Omega_{l}^{\varepsilon}\right)^{3}, l=1,2$ we use the notation $\mathbf{v}_{l}^{\varepsilon}$ for the trace of $\mathbf{v}_{l}^{\varepsilon}$ on $\partial \Omega_{l}^{\varepsilon}$ and we denote by $\mathbf{v}_{l \nu}^{\varepsilon}$ and $\mathbf{v}_{l \tau}^{\varepsilon}$ the normal and the tangential components of $\mathbf{v}_{l}^{\varepsilon}$ on the boundary, given by

$$
\mathbf{v}_{l \nu}^{\varepsilon}=\mathbf{v}_{l}^{\varepsilon} . \nu_{l}, \quad \mathbf{v}_{l \tau}^{\varepsilon}=\mathbf{v}_{l}^{\varepsilon}-\mathbf{v}_{l \nu}^{\varepsilon} \nu_{l}, \text { with } \nu=\nu_{1}=-\nu_{2}
$$

For a regular stress field $\sigma_{l}^{\varepsilon}$, the application of its trace on the boundary to $\nu_{l}$ is the Cauchy stress vector $\sigma_{l}^{\varepsilon} \nu_{l}$. We define, similarly, the normal and tangential components of the stress on the boundary by the formulas

$$
\sigma_{l \nu}^{\varepsilon}=\left(\sigma_{l}^{\varepsilon} \nu_{l}\right) \cdot \nu_{l}, \quad \sigma_{l \tau}^{\varepsilon}=\sigma_{l}^{\varepsilon} \nu_{l}-\sigma_{l \nu}^{\varepsilon} . \nu_{l} .
$$

To describe the boundary conditions, let us first introduce a vector function $g_{l}=\left(g_{l i}\right)_{1 \leq i \leq 3}, l=1,2$, such that

$$
\int_{\partial \Omega_{l}^{\varepsilon}} g_{l} \cdot \nu_{l} d s=0, l=1,2
$$

We assume that the function $g_{l}$ is in $H^{\frac{1}{2}}\left(\partial \Omega_{l}^{\varepsilon}\right)^{3}$, the space of traces of functions from $H^{1}\left(\Omega_{l}^{\varepsilon}\right)^{3}$ on $\partial \Omega_{l}^{\varepsilon}$.

Due to $\int_{\partial \Omega_{l}^{\varepsilon}} g_{l} \cdot \nu_{l} d s=0, l=1,2$, it is well known that there exists a function $G^{\varepsilon}=\left(G_{l}^{\varepsilon}\right)_{1 \leq l \leq 2}$ (see. [4]) such that

$$
G_{l}^{\varepsilon} \in H^{1}\left(\Omega_{l}^{\varepsilon}\right)^{3} \text { with } G_{l}^{\varepsilon}=g_{l} \text { on } \partial \Omega_{l}
$$

\section{The model and its variational formulation}

The physical setting is as follows. We consider two elastic bodies that occupy the domains $\Omega_{1}^{\varepsilon}$ and $\Omega_{2}^{\varepsilon}$. The two bodies are in bilateral, frictionless, contact along the common part $\omega$.

We denote by $\mathbf{u}_{l}^{\varepsilon}=\left(u_{l i}^{\varepsilon}\right)_{1 \leq i \leq 3}, l=1,2$ the displacement vectors, by $\sigma_{l}^{\varepsilon}=\left(\sigma_{l i j}^{\varepsilon}\right)_{1 \leq i, j \leq 3}, l=1,2$, the stress tensor and by $d_{i j}\left(\mathbf{u}_{l}^{\varepsilon}\right)$ the linearized strain tensors. We model the materials with linear elastic constitutive laws

$$
\sigma_{l i j}^{\varepsilon}\left(\mathbf{u}_{l}^{\varepsilon}\right)=2 \mu_{l} d_{i j}\left(\mathbf{u}_{l}^{\varepsilon}\right)+\lambda_{l} d_{k k}\left(\mathbf{u}_{l}^{\varepsilon}\right) \delta_{i j}, 1 \leq i, j, k \leq 3, l=1,2,
$$

where, $\mu_{l}, \lambda_{l}$ denote the Lamé coefficients $\delta=\left(\delta_{i j}\right)$ the identity tensor. 
- The upper surface $\Gamma_{l}^{\varepsilon}, l=1,2$ being assumed to be fixed so:

$$
\mathbf{u}_{l}^{\varepsilon}=0, l=1,2 .
$$

- On $\Gamma_{L_{l}}^{\varepsilon}, l=1,2$ the displacement is known and parallel to the $\omega$-plane:

$$
\mathbf{u}_{l}^{\varepsilon}=g_{l} \text { with } g_{l 3}=0, l=1,2 \text {. }
$$

- We describe now the conditions on the common surface $\omega$. We assume that the contact is bilateral, i.e.,

$$
\mathbf{u}_{1 \nu}^{\varepsilon}+\mathbf{u}_{2 \nu}^{\varepsilon}=0 \text { on } \omega
$$

Therefore,

$$
\nu_{1}=-\nu_{2} \text { and } \sigma_{1}^{\varepsilon} \cdot \nu_{1}=-\sigma_{2}^{\varepsilon} \cdot \nu_{2} \text { on } \omega
$$

Consequently,

$$
\sigma_{\nu}^{\varepsilon}=\sigma_{1 \nu}^{\varepsilon}=\sigma_{2 \nu}^{\varepsilon} \text { and } \sigma_{\tau}^{\varepsilon}=\sigma_{1 \tau}^{\varepsilon}=-\sigma_{2 \tau}^{\varepsilon} \text { on } \omega
$$

However, the tangential velocity is unknown and satisfies the Tresca boundary condition:

$$
\left\{\begin{array}{l}
\left|\sigma_{\tau}^{\varepsilon}\right|<k^{\varepsilon} \Longrightarrow \mathbf{u}_{1 \tau}^{\varepsilon}-\mathbf{u}_{2 \tau}^{\varepsilon}=s \\
\left|\sigma_{\tau}^{\varepsilon}\right|=k^{\varepsilon} \Longrightarrow \exists \lambda \geq 0, \mathbf{u}_{1 \tau}^{\varepsilon}-\mathbf{u}_{2 \tau}^{\varepsilon}=s-\lambda \sigma_{\tau}^{\varepsilon}, \quad \text { on } \omega
\end{array}\right.
$$

The steady-state transmission problem for the elastic bodies is given by the following mechanical problem.

Problem $P^{\varepsilon}$. Find a displacement field $\mathbf{u}_{l}^{\varepsilon}=\left(u_{l i}^{\varepsilon}\right)_{1 \leq i \leq 3}: \Omega_{l}^{\varepsilon} \longrightarrow \mathbb{R}^{3}, l=1,2$ such that

$$
\begin{gathered}
\operatorname{div} \sigma_{1}^{\varepsilon}+\mathbf{f}_{1}^{\varepsilon}=0 \text { in } \Omega_{1}^{\varepsilon}, \\
\operatorname{div} \sigma_{2}^{\varepsilon}+\mathbf{f}_{2}^{\varepsilon}=0 \text { in } \Omega_{2}^{\varepsilon}, \\
\sigma_{1}^{\varepsilon}\left(\mathbf{u}_{1}^{\varepsilon}\right)=2 \mu_{1} d\left(\mathbf{u}_{1}^{\varepsilon}\right)+\lambda_{1} d_{k k}\left(\mathbf{u}_{1}^{\varepsilon}\right) \delta \text { in } \Omega_{1}^{\varepsilon}, \\
\sigma_{2}^{\varepsilon}\left(\mathbf{u}_{2}^{\varepsilon}\right)=2 \mu_{2} d\left(\mathbf{u}_{2}^{\varepsilon}\right)+\lambda_{2} d_{k k}\left(\mathbf{u}_{2}^{\varepsilon}\right) \delta \text { in } \Omega_{2}^{\varepsilon}, \\
\mathbf{u}_{1}^{\varepsilon}=0 \text { on } \Gamma_{1}^{\varepsilon}, \\
\mathbf{u}_{2}^{\varepsilon}=0 \text { on } \Gamma_{2}^{\varepsilon}, \\
\mathbf{u}_{1}^{\varepsilon}=g_{1} \text { on } \Gamma_{L_{1}}^{\varepsilon}, \\
\mathbf{u}_{2}^{\varepsilon}=g_{2} \text { on } \Gamma_{L_{2}}^{\varepsilon}, \\
\mathbf{u}_{1}^{\varepsilon} \cdot \nu-\mathbf{u}_{2}^{\varepsilon} \cdot \nu=0 \text { on } \omega, \\
\sigma_{1}^{\varepsilon} \cdot \nu-\sigma_{2}^{\varepsilon} . \nu=0 \text { on } \omega, \\
\left\{\begin{array}{l}
\mathbf{u}_{1 \tau}^{\varepsilon}-\mathbf{u}_{2 \tau}^{\varepsilon}=s \\
\left|\sigma_{\tau}^{\varepsilon}\right|<k^{\varepsilon}, \mathbf{u}_{1 \tau}^{\varepsilon}-\mathbf{u}_{2 \tau}^{\varepsilon}=s-\lambda \sigma_{\tau}^{\varepsilon}, \quad \text { on } \omega . \\
\left|\sigma_{\tau}^{\varepsilon}\right|=k^{\varepsilon} \Longrightarrow \quad
\end{array}\right.
\end{gathered}
$$


Lemma 3.1 ([1]). The condition of Tresca (3.11) is equivalent to

$$
\left(\mathbf{u}_{1}^{\varepsilon}-\mathbf{u}_{2}^{\varepsilon}-s\right) \sigma_{\tau}^{\varepsilon}+k^{\varepsilon}\left|\left(\mathbf{u}_{1}^{\varepsilon}-\mathbf{u}_{2}^{\varepsilon}-s\right)\right|=0 \text { on } \omega .
$$

Theorem 3.2. Let $\left(\mathbf{u}_{1}^{\varepsilon}, \mathbf{u}_{2}^{\varepsilon}\right)$ be the solution of (3.1)-(3.11), then it checks the following variational problem

$$
\left\{\begin{array}{l}
\text { Find }\left(\mathbf{u}_{1}^{\varepsilon}, \mathbf{u}_{2}^{\varepsilon}\right) \in V^{\varepsilon} \text { such that } \\
\mathcal{A}\left(\left(\mathbf{u}_{1}^{\varepsilon}, \mathbf{u}_{2}^{\varepsilon}\right),\left(\varphi_{1}-\mathbf{u}_{1}^{\varepsilon}, \varphi_{2}-\mathbf{u}_{2}^{\varepsilon}\right)\right)+J^{\varepsilon}\left(\varphi_{1}, \varphi_{2}\right)-J^{\varepsilon}\left(\mathbf{u}_{1}^{\varepsilon}, \mathbf{u}_{2}^{\varepsilon}\right) \\
\quad \geq \int_{\Omega_{1}^{\varepsilon}} \mathbf{f}_{1}^{\varepsilon} \cdot\left(\varphi_{1}-\mathbf{u}_{1}^{\varepsilon}\right) d x+\int_{\Omega_{2}^{\varepsilon}} \mathbf{f}_{2}^{\varepsilon} \cdot\left(\varphi_{2}-\mathbf{u}_{2}^{\varepsilon}\right) d x \quad \forall\left(\varphi_{1}, \varphi_{2}\right) \in V^{\varepsilon}
\end{array}\right.
$$

where

$$
\begin{aligned}
\mathcal{A}\left(\left(\mathbf{u}_{1}, \mathbf{u}_{2}\right),\left(\varphi_{1}, \varphi_{2}\right)\right) & =\sum_{1 \leq l \leq 2}\left\{2 \mu_{l} \int_{\Omega_{l}^{\varepsilon}} d_{i j}\left(\mathbf{u}_{l}\right) d_{i j}\left(\varphi_{l}\right) d x+\lambda_{l} \int_{\Omega_{l}^{\varepsilon}} \operatorname{div}\left(\mathbf{u}_{l}\right) \operatorname{div}\left(\varphi_{l}\right) d x\right\}, \\
J^{\varepsilon}\left(\mathbf{v}_{1}, \mathbf{v}_{2}\right) & =\int_{\omega} k^{\varepsilon}\left|\left(\mathbf{v}_{1 \tau}-\mathbf{v}_{2 \tau}-s\right)\right| d x^{\prime} .
\end{aligned}
$$

Proof. Suppose $\left(\mathbf{u}_{1}^{\varepsilon}, \mathbf{u}_{2}^{\varepsilon}\right)$ be the solution of (3.1)-(3.11) that is sufficiently regular. Multiplying equation (3.1) by $\left(\varphi_{1}-\mathbf{u}_{1}^{\varepsilon}\right)$ and equation (3.2) by $\left(\varphi_{2}-\mathbf{u}_{2}^{\varepsilon}\right)$, where $\left(\varphi_{1}, \varphi_{2}\right) \in V^{\varepsilon}$, and we use Green's formula on each subdomain $\Omega_{l}^{\varepsilon}, l=1,2$, we get

$$
\begin{aligned}
& \int_{\Omega_{1}^{\varepsilon}} \sigma_{1 i j}^{\varepsilon} \frac{\partial}{\partial x_{j}}\left(\varphi_{1 i}-u_{1 i}^{\varepsilon}\right) d x+\int_{\Omega_{2}^{\varepsilon}} \sigma_{2 i j}^{\varepsilon} \frac{\partial}{\partial x_{j}}\left(\varphi_{2 i}-u_{2 i}^{\varepsilon}\right) d x \\
& \quad-\int_{\partial \Omega_{1}^{\varepsilon}} \sigma_{1 i j}^{\varepsilon} \nu_{1 j}\left(\varphi_{1 i}-u_{1 i}^{\varepsilon}\right) d s-\int_{\partial \Omega_{2}^{\varepsilon}} \sigma_{2 i j}^{\varepsilon} \nu_{2 j}\left(\varphi_{2 i}-u_{2 i}^{\varepsilon}\right) d s \\
& =\int_{\Omega_{1}^{\varepsilon}} f_{1 i}^{\varepsilon}\left(\varphi_{1 i}-u_{1 i}^{\varepsilon}\right) d x+\int_{\Omega_{2}^{\varepsilon}} f_{2 i}^{\varepsilon}\left(\varphi_{2 i}-u_{2 i}^{\varepsilon}\right) d x \forall\left(\varphi_{1}, \varphi_{2}\right) \in V^{\varepsilon} .
\end{aligned}
$$

According to the boundary conditions (3.5) - (3.10), we find

$$
\begin{aligned}
\int_{\partial \Omega_{1}^{\varepsilon}} & \sigma_{1 i j}^{\varepsilon} \nu_{1 j}\left(\varphi_{1 i}-u_{1 i}^{\varepsilon}\right) d s+\int_{\partial \Omega_{2}^{\varepsilon}} \sigma_{2 i j}^{\varepsilon} \nu_{2 j}\left(\varphi_{2 i}-u_{2 i}^{\varepsilon}\right) d s \\
= & \int_{\omega} \sigma_{1}^{\varepsilon} \nu_{1}\left(\varphi_{1}-\mathbf{u}_{1}^{\varepsilon}\right) d x^{\prime}+\int_{\omega} \sigma_{2}^{\varepsilon} \nu_{2}\left(\varphi_{2}-\mathbf{u}_{2}^{\varepsilon}\right) d x^{\prime}, \\
= & \int_{\omega} \sigma_{\tau}^{\varepsilon}\left[\left(\varphi_{1 \tau}-\mathbf{u}_{1 \tau}^{\varepsilon}\right)-\left(\varphi_{2 \tau}-\mathbf{u}_{2 \tau}^{\varepsilon}\right)\right] d x^{\prime}+\int_{\omega} \sigma_{\nu}^{\varepsilon}\left[\left(\varphi_{1 \tau}-\mathbf{u}_{1 \tau}^{\varepsilon}\right) \cdot \nu-\left(\varphi_{2 \tau}-\mathbf{u}_{2 \tau}^{\varepsilon}\right) \cdot \nu\right] d x^{\prime}, \\
= & \int_{\omega} \sigma_{\tau}^{\varepsilon}\left[\left(\varphi_{1 \tau}-\varphi_{2 \tau}\right)-\left(\mathbf{u}_{1 \tau}^{\varepsilon}-\mathbf{u}_{2 \tau}^{\varepsilon}\right)\right] d x^{\prime} .
\end{aligned}
$$

Therefore,

$$
\begin{aligned}
& \int_{\Omega_{1}^{\varepsilon}} \sigma_{1 i j}^{\varepsilon} \frac{\partial}{\partial x_{j}}\left(\varphi_{1 i}-u_{1 i}^{\varepsilon}\right) d x+\int_{\Omega_{2}^{\varepsilon}} \sigma_{2 i j}^{\varepsilon} \frac{\partial}{\partial x_{j}}\left(\varphi_{2 i}-u_{2 i}^{\varepsilon}\right) d x+J^{\varepsilon}\left(\varphi_{1}, \varphi_{2}\right)-J^{\varepsilon}\left(\mathbf{u}_{1}^{\varepsilon}, \mathbf{u}_{2}^{\varepsilon}\right) \\
& \quad-\int_{\Omega_{1}^{\varepsilon}} f_{1 i}^{\varepsilon}\left(\varphi_{1 i}-u_{1 i}^{\varepsilon}\right) d x-\int_{\Omega_{2}^{\varepsilon}} f_{2 i}^{\varepsilon}\left(\varphi_{2 i}-u_{2 i}^{\varepsilon}\right) d x \\
& =\int_{\omega} \sigma_{\tau}^{\varepsilon}\left(\left(\varphi_{1 \tau}-\varphi_{2 \tau}-s\right)-\left(\mathbf{u}_{1 \tau}^{\varepsilon}-\mathbf{u}_{2 \tau}^{\varepsilon}-s\right)\right) d x^{\prime} \\
& \quad+\int_{\omega} k^{\varepsilon}\left(\left|\varphi_{1 \tau}-\varphi_{2 \tau}-s\right|-\left|\mathbf{u}_{1 \tau}^{\varepsilon}-\mathbf{u}_{2 \tau}^{\varepsilon}-s\right|\right) d x^{\prime}
\end{aligned}
$$

Using (3.12) we deduce directly the variational inequality 3.13 . 
The existence and uniqueness results of the weak solution to the problem $3.1-3.11$ is obtained in the following theorem.

Theorem 3.3. Assuming that $\left(\mathbf{f}_{1}^{\varepsilon}, \mathbf{f}_{2}^{\varepsilon}\right) \in L^{2}\left(\Omega_{1}^{\varepsilon}\right)^{3} \times L^{2}\left(\Omega_{2}^{\varepsilon}\right)^{3}, k^{\varepsilon} \in L^{2}(\omega)$, there exists a unique solution $\left(\mathbf{u}_{1}^{\varepsilon}, \mathbf{u}_{2}^{\varepsilon}\right) \in V^{\varepsilon}$ to 3.13 .

Proof. A bilinear form $\mathcal{A}(.,$.$) is coercive on V^{\varepsilon} \times V^{\varepsilon}$.

Indeed, let $\left(\mathbf{v}_{1}^{\varepsilon}, \mathbf{v}_{2}^{\varepsilon}\right)$ an element of $V^{\varepsilon}$. By Korn's inequality, we obtain

$$
\begin{aligned}
\mathcal{A}\left(\left(\mathbf{v}_{1}^{\varepsilon}, \mathbf{v}_{2}^{\varepsilon}\right),\left(\mathbf{v}_{1}^{\varepsilon}, \mathbf{v}_{2}^{\varepsilon}\right)\right) & \geq 2 \mu_{1} \int_{\Omega_{1}^{\varepsilon}} d_{i j}\left(\mathbf{v}_{1}^{\varepsilon}\right) d_{i j}\left(\mathbf{v}_{1}^{\varepsilon}\right) d x+2 \mu_{2} \int_{\Omega_{2}^{\varepsilon}} d_{i j}\left(\mathbf{v}_{2}^{\varepsilon}\right) d_{i j}\left(\mathbf{v}_{2}^{\varepsilon}\right) d x, \\
& \geq 2 C_{K}\left(\mu_{1}\left\|\mathbf{v}_{1}^{\varepsilon}\right\|_{V\left(\Omega_{1}^{\varepsilon}\right)}^{2}+\mu_{2}\left\|\mathbf{v}_{2}^{\varepsilon}\right\|_{V\left(\Omega_{2}^{\varepsilon}\right)}^{2}\right) \\
& \geq 2 \mu_{-} C_{k}\left\|\left(\mathbf{v}_{1}^{\varepsilon}, \mathbf{v}_{2}^{\varepsilon}\right)\right\|_{V^{\varepsilon}}^{2},
\end{aligned}
$$

where $C_{k}>0$, is the constant of Korn and $\mu_{-}=\min \left(\mu_{1}, \mu_{2}\right)$.

Therefore,

$$
\begin{aligned}
\left|\mathcal{A}\left(\left(\mathbf{u}_{1}^{\varepsilon}, \mathbf{u}_{2}^{\varepsilon}\right),\left(\mathbf{v}_{1}^{\varepsilon}, \mathbf{v}_{2}^{\varepsilon}\right)\right)\right| & \leq \sum_{1 \leq l \leq 2}\left(2 \mu_{l} \int_{\Omega_{l}^{\varepsilon}}\left|d_{i j}\left(\mathbf{u}_{l}^{\varepsilon}\right) d_{i j}\left(\mathbf{v}_{l}^{\varepsilon}\right)\right| d x+\lambda_{l} \int_{\Omega_{l}^{\varepsilon}}\left|\operatorname{div}\left(\mathbf{u}_{l}^{\varepsilon}\right) \operatorname{div}\left(\mathbf{v}_{l}^{\varepsilon}\right)\right| d x\right) \\
& \leq \sum_{1 \leq l \leq 2}\left(2 \mu_{l}\left\|\mathbf{u}_{l}^{\varepsilon}\right\|_{1, \Omega_{l}^{\varepsilon}}\left\|\mathbf{v}_{l}^{\varepsilon}\right\|_{1, \Omega_{l}^{\varepsilon}}+\lambda_{l}\left\|\mathbf{u}_{l}^{\varepsilon}\right\|_{1, \Omega_{l}^{\varepsilon}}\left\|\mathbf{v}_{l}^{\varepsilon}\right\|_{1, \Omega_{l}^{\varepsilon}}\right) \\
& \leq C\left\|\left(\mathbf{u}_{1}^{\varepsilon}, \mathbf{u}_{2}^{\varepsilon}\right)\right\|_{V^{\varepsilon}}\left\|\left(\mathbf{v}_{1}^{\varepsilon}, \mathbf{v}_{2}^{\varepsilon}\right)\right\|_{V^{\varepsilon}},
\end{aligned}
$$

where, $C=\max _{1 \leq l \leq 2}\left(2 \mu_{l}+\lambda_{l}\right)$.

On the other hand, $J^{\varepsilon}$ is a convex and continuous functional on $V^{\varepsilon}$. Then the existence and uniqueness of $\left(\mathbf{u}_{1}^{\varepsilon}, \mathbf{u}_{2}^{\varepsilon}\right)$ in $V^{\varepsilon}$ satisfying the variational inequality (3.13).

\section{The problem in a fixed domain}

This section is devoted to the study of a priori estimates on the displacement $u^{\varepsilon}=\left(\mathbf{u}_{1}^{\varepsilon}, \mathbf{u}_{2}^{\varepsilon}\right)$, solution of our variational problem. For the asymptotic analysis of problem (3.1)-(3.11), we use the approach which consist in transposing the problem initially posed in the domain $\Omega_{l}^{\varepsilon}$ which depend on a small parameter $\varepsilon$ in an equivalent problem posed in the fixed domain $\Omega_{l}$ which is independent of $\varepsilon$. For that, we introduce a small change of the variable $z=\frac{x_{3}}{\varepsilon}$, so for $\left(x, x_{3}\right)$ in $\Omega_{l}^{\varepsilon}$ we have $(x, z)$ in

$$
\begin{aligned}
& \Omega_{1}=\left\{\left(x^{\prime}, z\right) \in \mathbb{R}^{3},\left(x^{\prime}, 0\right) \in \omega, \quad 0<z<h\left(x^{\prime}\right)\right\}, \\
& \Omega_{2}=\left\{\left(x^{\prime}, z\right) \in \mathbb{R}^{3},\left(x^{\prime}, 0\right) \in \omega, \quad-h\left(x^{\prime}\right)<z<0\right\} .
\end{aligned}
$$

We denote by $\partial \Omega_{l}=\bar{\omega} \cup \bar{\Gamma}_{l} \cup \bar{\Gamma}_{L_{l}}, l=1,2$ its boundary, then we define the following functions in $\Omega_{l}$

$$
\left\{\begin{array}{l}
\hat{u}_{l i}^{\varepsilon}\left(x^{\prime}, z\right)=u_{l i}^{\varepsilon}\left(x^{\prime}, x_{3}\right), i=1,2 \\
\hat{u}_{l 3}^{\varepsilon}\left(x^{\prime}, z\right)=\varepsilon^{-1} u_{l 3}^{\varepsilon}\left(x^{\prime}, x_{3}\right), l=1,2
\end{array}\right.
$$

For the data of the problem (3.1)-(3.11), we suppose that they depend on $\varepsilon$ in a following manner:

$$
\left\{\begin{array}{l}
\hat{\mathbf{f}}_{l}\left(x^{\prime}, z\right)=\varepsilon^{2} \mathbf{f}_{l}^{\varepsilon}\left(x^{\prime}, x_{3}\right), \\
\hat{k}=\varepsilon k^{\varepsilon}, \\
\hat{g}_{l}\left(x^{\prime}, z\right)=g_{l}^{\varepsilon}\left(x^{\prime}, x_{3}\right),
\end{array}\right.
$$


with $\hat{\mathbf{f}}_{l}, \hat{k}$ and $\hat{g}_{l}$ independent of $\varepsilon$.

The vector $G_{l}^{\varepsilon}$ introduced in Section 2 will be defined as follows

$$
\left\{\begin{array}{l}
\hat{G}_{l i}\left(x^{\prime}, z\right)=G_{l i}^{\varepsilon}\left(x^{\prime}, x_{3}\right), i=1,2, \quad l=1,2 . \\
\hat{G}_{l 3}\left(x^{\prime}, z\right)=\varepsilon^{-1} G_{l 3}^{\varepsilon}\left(x^{\prime}, x_{3}\right),
\end{array}\right.
$$

Now we introduce the functional framework on $\Omega_{1} \cup \Omega_{2}$. For this, we note

$$
\begin{aligned}
V\left(\Omega_{l}\right) & =\left\{\widehat{\mathbf{v}} \in H^{1}\left(\Omega_{l}\right)^{3}: \widehat{\mathbf{v}}=0 \text { on } \Gamma_{l} \cup \Gamma_{L_{l}}\right\}, \\
V & =\left\{\left(\widehat{\mathbf{v}}_{1}, \widehat{\mathbf{v}}_{2}\right) \in V\left(\Omega_{1}\right) \times V\left(\Omega_{2}\right): \widehat{\mathbf{v}}_{1} \cdot \nu-\widehat{\mathbf{v}}_{2} \cdot \nu=0 \text { on } \omega\right\}, \\
H_{z}\left(\Omega_{l}\right) & =\left\{\widehat{\mathbf{v}}_{l}=\left(\widehat{\mathbf{v}}_{l 1}, \widehat{\mathbf{v}}_{l 2}\right) \in L^{2}\left(\Omega_{l}\right)^{2}: \frac{\partial \widehat{\mathbf{v}}_{l i}}{\partial z} \in L^{2}\left(\Omega_{l}\right), i=1,2 \text { and } \widehat{\mathbf{v}}_{l}=0 \text { on } \Gamma_{l}\right\}, \\
H_{z} & =H_{z}\left(\Omega_{1}\right) \times H_{z}\left(\Omega_{2}\right), \\
\overline{V\left(\Omega_{l}\right)} & =\left\{\bar{\varphi}_{l} \in H^{1}\left(\Omega_{l}\right)^{2}: \bar{\varphi}_{l}=\left(\varphi_{l 1}, \varphi_{l 2}\right), \bar{\varphi}_{l i}=0 \text { on } \Gamma_{l} \cup \Gamma_{L_{l}} \text { for } i=1,2\right\} .
\end{aligned}
$$

$H_{z}\left(\Omega_{l}\right), l=1,2$, is a Banach space for the norm

$$
\begin{aligned}
\left\|\widehat{\mathbf{v}}_{l}\right\|_{H_{z}\left(\Omega_{l}\right)} & =\left\{\sum_{i=1}^{2}\left(\left\|\widehat{\mathbf{v}}_{l i}\right\|_{0, \Omega_{l}}^{2}+\left\|\frac{\partial \widehat{\mathbf{v}}_{l i}}{\partial z}\right\|_{0, \Omega_{l}}^{2}\right)\right\}^{\frac{1}{2}}, \\
\left\|\left(\widehat{\mathbf{v}}_{1}, \widehat{\mathbf{v}}_{2}\right)\right\|_{H_{z}} & =\left(\left\|\widehat{\mathbf{v}}_{1}\right\|_{H_{z}\left(\Omega_{1}\right)}^{2}+\left\|\widehat{\mathbf{v}}_{2}\right\|_{H_{z}\left(\Omega_{2}\right)}^{2}\right)^{\frac{1}{2}} .
\end{aligned}
$$

With these new notations, the variational problem $(3.13)$ is equivalent to the following problem:

$$
\left\{\begin{array}{l}
\text { Find }\left(\hat{\mathbf{u}}_{1}^{\varepsilon}, \hat{\mathbf{u}}_{2}^{\varepsilon}\right) \in V \text {, such that } \\
\begin{array}{rl}
\mathcal{A}\left(\left(\hat{\mathbf{u}}_{1}^{\varepsilon}, \hat{\mathbf{u}}_{2}^{\varepsilon}\right),\left(\hat{\varphi}_{1}-\hat{\mathbf{u}}_{1}^{\varepsilon}, \hat{\varphi}_{2}-\hat{\mathbf{u}}_{2}^{\varepsilon}\right)\right)+\hat{J}\left(\hat{\varphi}_{1}, \hat{\varphi}_{2}\right) \\
\quad-\hat{J}\left(\hat{\mathbf{u}}_{1}^{\varepsilon}, \hat{\mathbf{u}}_{2}^{\varepsilon}\right) \geq \sum_{i=1}^{2} \int_{\Omega_{1}^{\varepsilon}} \hat{\mathbf{f}}_{1 i}\left(\hat{\varphi}_{1 i}-\hat{\mathbf{u}}_{1 i}^{\varepsilon}\right) d x+\varepsilon \int_{\Omega_{1}^{\varepsilon}} \hat{\mathbf{f}}_{13}\left(\hat{\varphi}_{13}-\hat{\mathbf{u}}_{13}^{\varepsilon}\right) d x \\
\quad+\sum_{i=1}^{2} \int_{\Omega_{2}^{\varepsilon}} \hat{\mathbf{f}}_{2 i}\left(\hat{\varphi}_{2 i}-\hat{\mathbf{u}}_{2 i}^{\varepsilon}\right) d x+\varepsilon \int_{\Omega_{2}^{\varepsilon}} \hat{\mathbf{f}}_{23}\left(\hat{\varphi}_{23}-\hat{\mathbf{u}}_{23}^{\varepsilon}\right) d x \quad \forall\left(\hat{\varphi}_{1}, \hat{\varphi}_{2}\right) \in V,
\end{array}
\end{array}\right.
$$

where,

$$
\hat{J}\left(\hat{\varphi}_{1}, \hat{\varphi}_{2}\right)=\int_{\omega} \hat{k}\left|\left(\hat{\varphi}_{1 \tau}-\hat{\varphi}_{2 \tau}-s\right)\right| d x^{\prime}
$$

and

$$
\begin{aligned}
\mathcal{A}\left(\left(\hat{\mathbf{u}}_{1}^{\varepsilon}, \hat{\mathbf{u}}_{2}^{\varepsilon}\right),\left(\hat{\varphi}_{1}-\hat{\mathbf{u}}_{1}^{\varepsilon}, \hat{\varphi}_{2}-\hat{\mathbf{u}}_{2}^{\varepsilon}\right)\right) \\
=\varepsilon^{2} \sum_{1 \leq i, j, l \leq 2}\left\{\mu_{l} \int_{\Omega_{l}}\left(\frac{\partial \hat{u}_{l i}^{\varepsilon}}{\partial x_{j}}+\frac{\partial \hat{u}_{l j}^{\varepsilon}}{\partial x_{i}}\right) \frac{\partial}{\partial x_{j}}\left(\hat{\varphi}_{l i}-\hat{u}_{l i}^{\varepsilon}\right) d x^{\prime} d z\right\} \\
\quad+\sum_{1 \leq i, l \leq 2}\left\{\mu_{l} \int_{\Omega_{l}}\left(\frac{\partial \hat{u}_{l i}^{\varepsilon}}{\partial z}+\varepsilon^{2} \frac{\partial \hat{u}_{l 3}^{\varepsilon}}{\partial x_{i}}\right)\left[\frac{\partial}{\partial z}\left(\hat{\varphi}_{l i}-\hat{u}_{l i}^{\varepsilon}\right)+\varepsilon^{2} \frac{\partial}{\partial x_{i}}\left(\hat{\varphi}_{l 3}-\hat{u}_{l 3}^{\varepsilon}\right)\right] d x^{\prime} d z\right\} \\
\quad+\sum_{1 \leq l \leq 2}\left\{2 \mu_{l} \varepsilon^{2} \int_{\Omega_{l}} \frac{\partial \hat{u}_{l 3}^{\varepsilon}}{\partial z} \cdot \frac{\partial}{\partial z}\left(\hat{\varphi}_{l 3}-\hat{u}_{l 3}^{\varepsilon}\right) d x d z+\lambda_{l} \varepsilon^{2} \int_{\Omega_{l}} \operatorname{div}\left(\hat{u}_{l}^{\varepsilon}\right) \operatorname{div}\left(\hat{\varphi}_{l}-\hat{u}_{l}^{\varepsilon}\right) d x^{\prime} d z\right\} .
\end{aligned}
$$


In the next, we will obtain estimates on $\hat{u}^{\varepsilon}=\left(\hat{\mathbf{u}}_{1}^{\varepsilon}, \hat{\mathbf{u}}_{2}^{\varepsilon}\right)$. These estimates will be useful in order to prove the convergence of $\hat{u}^{\varepsilon}$ toward the expected function. For this, we introduce some results which will be used in the next. The detailed description can be found in [8].

$$
\begin{aligned}
\left\|\nabla \widehat{\varphi}_{l}\right\|_{0, \Omega_{l}^{\varepsilon}} & \leq C\left\|D\left(\widehat{\varphi}_{l}\right)\right\|_{L^{2}\left(\Omega_{l}\right)}, l=1,2, \text { for }\left(\widehat{\varphi}_{1}, \widehat{\varphi}_{2}\right) \in V \text { (Korn inequality) }, \\
\left\|\left(\hat{u}_{1 i}^{\varepsilon}, \hat{u}_{2 i}^{\varepsilon}\right)\right\|_{0, \Omega_{1} \times \Omega_{2}} & \leq h^{*}\left\|\left(\frac{\partial \hat{u}_{1 i}^{\varepsilon}}{\partial z}, \frac{\partial \hat{u}_{2 i}^{\varepsilon}}{\partial z}\right)\right\|_{0, \Omega_{1} \times \Omega_{2}}, i=1,2 \text { (Poincaré inequality), } \\
a b & \leq \frac{a^{r}}{r}+\frac{b^{r^{\prime}}}{r^{\prime}} \forall(a, b) \in \mathbb{R}^{2}, \text { (Young inequality). }
\end{aligned}
$$

Theorem 4.1. Assuming that $\left(\hat{\mathbf{f}}_{1}, \hat{\mathbf{f}}_{2}\right) \in L^{2}\left(\Omega_{1}\right)^{3} \times L^{2}\left(\Omega_{2}\right)^{3}$, the friction coefficient $\hat{k}$ in $L^{\infty}(\omega)$, then there exists a constant $C>0$ independent of $\varepsilon$ such that

$$
\begin{aligned}
& \varepsilon^{2} \sum_{1 \leq i, j \leq 2}\left\|\left(\frac{\partial \hat{u}_{1 i}^{\varepsilon}}{\partial x_{j}}, \frac{\partial \hat{u}_{2 i}^{\varepsilon}}{\partial x_{j}}\right)\right\|_{0, \Omega_{1} \times \Omega_{2}}^{2}+\varepsilon^{2}\left\|\left(\frac{\partial \hat{u}_{13}^{\varepsilon}}{\partial z}, \frac{\partial \hat{u}_{23}^{\varepsilon}}{\partial z}\right)\right\|_{0, \Omega_{1} \times \Omega_{2}}^{2} \\
& \quad+\sum_{1 \leq i \leq 2}\left(\left\|\left(\frac{\partial \hat{u}_{1 i}^{\varepsilon}}{\partial z}, \frac{\partial \hat{u}_{2 i}^{\varepsilon}}{\partial z}\right)\right\|_{0, \Omega_{1} \times \Omega_{2}}^{2}+\varepsilon^{4}\left\|\left(\frac{\partial \hat{u}_{13}^{\varepsilon}}{\partial x_{i}}, \frac{\partial \hat{u}_{23}^{\varepsilon}}{\partial x_{i}}\right)\right\|_{0, \Omega_{1} \times \Omega_{2}}^{2}\right) \leq C .
\end{aligned}
$$

Proof. Let $\left(\mathbf{u}_{1}^{\varepsilon}, \mathbf{u}_{2}^{\varepsilon}\right)$ be a solution to the problem (3.13), we deduce

$$
\begin{aligned}
& \mathcal{A}\left(\left(\mathbf{u}_{1}^{\varepsilon}, \mathbf{u}_{2}^{\varepsilon}\right),\left(\varphi_{1}-\mathbf{u}_{1}^{\varepsilon}, \varphi_{2}-\mathbf{u}_{2}^{\varepsilon}\right)\right)+J^{\varepsilon}\left(\varphi_{1}, \varphi_{2}\right)-J^{\varepsilon}\left(\mathbf{u}_{1}^{\varepsilon}, \mathbf{u}_{2}^{\varepsilon}\right) \\
& \quad \geq \int_{\Omega_{1}^{\varepsilon}} \mathbf{f}_{1}^{\varepsilon}\left(\varphi_{1}-\mathbf{u}_{1}^{\varepsilon}\right) d x+\int_{\Omega_{2}^{\varepsilon}} \mathbf{f}_{2}^{\varepsilon}\left(\varphi_{2}-\mathbf{u}_{2}^{\varepsilon}\right) d x \quad \forall\left(\varphi_{1}, \varphi_{2}\right) \in V^{\varepsilon} .
\end{aligned}
$$

As $J^{\varepsilon}\left(\mathbf{u}_{1}^{\varepsilon}, \mathbf{u}_{2}^{\varepsilon}\right) \geq 0$ is positive, we have

$$
\begin{aligned}
\mathcal{A}\left(\left(\mathbf{u}_{1}^{\varepsilon}, \mathbf{u}_{2}^{\varepsilon}\right),\left(\mathbf{u}_{1}^{\varepsilon}, \mathbf{u}_{2}^{\varepsilon}\right)\right) \leq & \mathcal{A}\left(\left(\mathbf{u}_{1}^{\varepsilon}, \mathbf{u}_{2}^{\varepsilon}\right),\left(\varphi_{1}, \varphi_{2}\right)\right)+J^{\varepsilon}\left(\varphi_{1}, \varphi_{2}\right)+\int_{\Omega_{1}^{\varepsilon}} \mathbf{f}_{1}^{\varepsilon} \mathbf{u}_{1}^{\varepsilon} d x \\
& +\int_{\Omega_{2}^{\varepsilon}} \mathbf{f}_{2}^{\varepsilon} \mathbf{u}_{2}^{\varepsilon} d x-\int_{\Omega_{1}^{\varepsilon}} \mathbf{f}_{1}^{\varepsilon} \varphi_{1} d x-\int_{\Omega_{2}^{\varepsilon}} \mathbf{f}_{2}^{\varepsilon} \varphi_{2} d x \quad \forall\left(\varphi_{1}, \varphi_{2}\right) \in V^{\varepsilon} .
\end{aligned}
$$

From Korn's inequality, there exists a constant $C_{K}>0$ independent of $\varepsilon$, such that

$$
C_{K}\left(2 \mu_{1}\left\|\nabla \mathbf{u}_{1}^{\varepsilon}\right\|_{0, \Omega_{1}^{\varepsilon}}^{2}+2 \mu_{2}\left\|\nabla \mathbf{u}_{2}^{\varepsilon}\right\|_{0, \Omega_{2}^{\varepsilon}}^{2}\right) \leq \mathcal{A}\left(\left(\mathbf{u}_{1}^{\varepsilon}, \mathbf{u}_{2}^{\varepsilon}\right),\left(\mathbf{u}_{1}^{\varepsilon}, \mathbf{u}_{2}^{\varepsilon}\right)\right) .
$$

Similarly, using Hölder and Young inequalities, we get

$$
\begin{aligned}
\mathcal{A}\left(\left(\mathbf{u}_{1}^{\varepsilon}, \mathbf{u}_{2}^{\varepsilon}\right),\left(\varphi_{1}, \varphi_{2}\right)\right) \leq & \sum_{1 \leq l \leq 2}\left\{\int_{\Omega_{l}^{\varepsilon}} 2 \mu_{l}\left|d_{i j}\left(\mathbf{u}_{l}^{\varepsilon}\right)\right|\left|d_{i j}\left(\varphi_{l}\right)\right| d x+\lambda_{l} \int_{\Omega_{l}^{\varepsilon}}\left|\operatorname{div}\left(\mathbf{u}_{l}^{\varepsilon}\right)\right|\left|\operatorname{div}\left(\varphi_{l}\right)\right| d x\right\} \\
\leq & \sum_{1 \leq l \leq 2}\left\{\left(\int_{\Omega_{l}^{\varepsilon}} \sqrt{\frac{\mu_{l} C_{K}}{2}}\left|d_{i j}\left(\mathbf{u}_{l}^{\varepsilon}\right)\right|^{2} d x\right)^{\frac{1}{2}}\left(\int_{\Omega_{l}^{\varepsilon}} \frac{2 \sqrt{2 \mu_{l}}}{\sqrt{C_{K}}}\left|d_{i j}\left(\varphi_{l}\right)\right|^{2} d x\right)^{\frac{1}{2}}\right\} \\
& +\sum_{1 \leq l \leq 2}\left\{\left(\int_{\Omega_{l}^{\varepsilon}} \frac{\sqrt{\mu_{l} C_{K}}}{2}\left|\operatorname{div}\left(\mathbf{u}_{l}^{\varepsilon}\right)\right|^{2} d x\right)^{\frac{1}{2}}\left(\int_{\Omega_{l}^{\varepsilon}} \frac{2 \lambda_{l}}{\sqrt{\mu_{l} C_{K}}}\left|\operatorname{div}\left(\varphi_{l}\right)\right|^{2} d x\right)^{\frac{1}{2}}\right\}
\end{aligned}
$$




$$
\begin{aligned}
\leq & \sum_{1 \leq l \leq 2}\left(\frac{\mu_{l} C_{K}}{4}\left\|d_{i j}\left(\mathbf{u}_{l}^{\varepsilon}\right)\right\|_{L^{2}\left(\Omega_{l}^{\varepsilon}\right)}^{2}+\frac{4 \mu_{l}}{C_{K}}\left\|d_{i j}\left(\varphi_{l}\right)\right\|_{L^{2}\left(\Omega_{l}^{\varepsilon}\right)}^{2}\right) \\
& +\sum_{1 \leq l \leq 2}\left(\frac{\mu_{l} C_{K}}{8} \int_{\Omega_{l}^{\varepsilon}}\left|\operatorname{div}\left(\mathbf{u}_{l}^{\varepsilon}\right)\right|^{2} d x+\frac{2\left(\lambda_{l}\right)^{2}}{\mu_{l} C_{K}} \int_{\Omega_{l}^{\varepsilon}}\left|\operatorname{div}\left(\varphi_{l}\right)\right|^{2} d x\right),
\end{aligned}
$$

and as $\sum_{1 \leq i, j \leq 2}\left|d_{i j}(\mathbf{v})\right|^{2} \leq|\nabla \mathbf{v}|^{2}$ and $|\operatorname{div}(\mathbf{v})|^{2} \leq|\nabla \mathbf{v}|^{2}$, we obtain

$$
\begin{aligned}
\mathcal{A}\left(\left(\mathbf{u}_{1}^{\varepsilon}, \mathbf{u}_{2}^{\varepsilon}\right),\left(\varphi_{1}, \varphi_{2}\right)\right) \leq \sum_{1 \leq l \leq 2} & \left\{\frac{\mu_{l} C_{K}}{4}\left\|\nabla \mathbf{u}_{l}^{\varepsilon}\right\|_{0, \Omega_{l}^{\varepsilon}}^{2}+\frac{4 \mu_{l}}{C_{K}}\left\|\nabla \varphi_{l}\right\|_{0, \Omega_{l}^{\varepsilon}}^{2}\right. \\
& \left.+\frac{\mu_{l} C_{K}}{8}\left\|\nabla \mathbf{u}_{l}^{\varepsilon}\right\|_{0, \Omega_{l}^{\varepsilon}}^{2}+\frac{2\left(\lambda_{l}\right)^{2}}{\mu_{l} C_{K}}\left\|\nabla \varphi_{l}\right\|_{0, \Omega_{l}^{\varepsilon}}^{2}\right\} .
\end{aligned}
$$

The analogue of (4.8) gives:

$$
\begin{aligned}
\left|\int_{\Omega_{1}^{\varepsilon}} \mathbf{f}_{1}^{\varepsilon} \mathbf{u}_{1}^{\varepsilon} d x+\int_{\Omega_{2}^{\varepsilon}} \mathbf{f}_{2}^{\varepsilon} \mathbf{u}_{2}^{\varepsilon} d x\right| \leq \sum_{1 \leq l \leq 2}\left(\frac{\mu_{l} C_{K}}{2}\left\|\nabla \mathbf{u}_{l}^{\varepsilon}\right\|_{0, \Omega_{l}^{\varepsilon}}^{2}+\frac{\left(\varepsilon h^{*}\right)^{2}}{2 \mu_{l} C_{K}}\left\|\nabla \mathbf{f}_{l}^{\varepsilon}\right\|_{0, \Omega_{l}^{\varepsilon}}^{2}\right), \\
\left|\int_{\Omega_{1}^{\varepsilon}} \mathbf{f}_{1}^{\varepsilon} \varphi_{1} d x+\int_{\Omega_{2}^{\varepsilon}} \mathbf{f}_{2}^{\varepsilon} \varphi_{2} d x\right| \leq \sum_{1 \leq l \leq 2}\left(\frac{\mu_{l} C_{K}}{2}\left\|\nabla \varphi_{l}\right\|_{0, \Omega_{l}^{\varepsilon}}^{2}+\frac{\left(\varepsilon h^{*}\right)^{2}}{2 \mu_{l} C_{K}}\left\|\nabla \mathbf{f}_{l}^{\varepsilon}\right\|_{0, \Omega_{l}^{\varepsilon}}^{2}\right) .
\end{aligned}
$$

Using (4.7)-4.10 and choosing $\varphi_{1}=G_{1}^{\varepsilon}$ and $\varphi_{2}=G_{2}^{\varepsilon}$, the variational inequality 4.6 is as following:

$$
\begin{aligned}
\sum_{1 \leq l \leq 2} 2 \mu_{l} C_{K}\left\|\nabla \mathbf{u}_{l}^{\varepsilon}\right\|_{0, \Omega_{l}^{\varepsilon}}^{2} \leq & \mathcal{A}\left(\left(\mathbf{u}_{1}^{\varepsilon}, \mathbf{u}_{2}^{\varepsilon}\right),\left(\mathbf{u}_{1}^{\varepsilon}, \mathbf{u}_{2}^{\varepsilon}\right)\right) \\
\leq & \sum_{1 \leq l \leq 2}\left\{\frac{\mu_{l} C_{K}}{4}\left\|\nabla \mathbf{u}_{l}^{\varepsilon}\right\|_{0, \Omega_{l}^{\varepsilon}}^{2}+\frac{4 \mu_{l}}{C_{K}}\left\|\nabla G_{l}^{\varepsilon}\right\|_{0, \Omega_{l}^{\varepsilon}}^{2}+\frac{\mu_{l} C_{K}}{8}\left\|\nabla \mathbf{u}_{l}^{\varepsilon}\right\|_{0, \Omega_{l}^{\varepsilon}}^{2}\right. \\
& +\frac{2\left(\lambda_{l}\right)^{2}}{\mu_{l} C_{K}}\left\|\nabla G_{l}^{\varepsilon}\right\|_{0, \Omega_{l}^{\varepsilon}}^{2}+\frac{\mu_{l} C_{K}}{2}\left\|\nabla \mathbf{u}_{l}^{\varepsilon}\right\|_{0, \Omega_{l}^{\varepsilon}}^{2}+\frac{\left(\varepsilon h^{*}\right)^{2}}{2 \mu_{l} C_{K}}\left\|\nabla \mathbf{f}_{l}^{\varepsilon}\right\|_{0, \Omega_{l}^{\varepsilon}}^{2} \\
& \left.+\frac{\mu_{l} C_{K}}{2}\left\|\nabla G_{l}^{\varepsilon}\right\|_{0, \Omega_{l}^{\varepsilon}}^{2}+\frac{\left(\varepsilon h^{*}\right)^{2}}{2 \mu_{l} C_{K}}\left\|\nabla \mathbf{f}_{l}^{\varepsilon}\right\|_{0, \Omega_{l}^{\varepsilon}}^{2}\right\},
\end{aligned}
$$

which implies

$$
\sum_{1 \leq l \leq 2} \frac{9}{8} \mu_{l} C_{K}\left\|\nabla \mathbf{u}_{l}^{\varepsilon}\right\|_{0, \Omega_{l}^{\varepsilon}}^{2} \leq \sum_{1 \leq l \leq 2}\left\{\frac{\left(\varepsilon h^{*}\right)^{2}}{\mu_{l} C_{K}}\left\|\nabla \mathbf{f}_{l}^{\varepsilon}\right\|_{0, \Omega_{l}^{\varepsilon}}^{2}+\left(\frac{2\left(\lambda_{l}\right)^{2}}{\mu_{l} C_{K}}+\frac{\mu_{l} C_{K}}{2}+\frac{4 \mu_{l}}{C_{K}}\right)\left\|\nabla G_{l}^{\varepsilon}\right\|_{0, \Omega_{l}^{\varepsilon}}^{2}\right\} .
$$

Multiplying (4.11) by $\varepsilon$ and for $0<\varepsilon<1$, we see that:

$$
\begin{aligned}
\frac{9}{8} \mu_{-} C_{K} \varepsilon\left(\left\|\nabla \mathbf{u}_{1}^{\varepsilon}\right\|_{0, \Omega_{1}^{\varepsilon}}^{2}+\left\|\nabla \mathbf{u}_{2}^{\varepsilon}\right\|_{0, \Omega_{2}^{\varepsilon}}^{2}\right) \leq & \frac{\left(h^{*}\right)^{2}}{\mu_{-} C_{K}}\left\|\nabla \hat{\mathbf{f}}_{1}\right\|_{0, \Omega_{1}}^{2}+\left(\frac{2\left(\lambda_{+}\right)^{2}}{\mu_{-} C_{K}}+\frac{\mu_{+} C_{K}}{2}+\frac{4 \mu_{+}}{C_{K}}\right)\left\|\nabla \hat{G}_{1}\right\|_{0, \Omega_{1}}^{2} \\
& +\frac{\left(h^{*}\right)^{2}}{\mu_{-} C_{K}}\left\|\nabla \hat{\mathbf{f}}_{2}\right\|_{0, \Omega_{2}}^{2}+\left(\frac{2\left(\lambda_{+}\right)^{2}}{\mu_{-} C_{K}}+\frac{\mu_{+} C_{K}}{2}+\frac{4 \mu_{+}}{C_{K}}\right)\left\|\nabla \hat{G}_{2}\right\|_{0, \Omega_{2}}^{2},
\end{aligned}
$$


with $\mu_{-}=\min \left(\mu_{1}, \mu_{2}\right), \mu_{+}=\max \left(\mu_{1}, \mu_{2}\right)$ and $\lambda_{+}=\max \left(\lambda_{1}, \lambda_{2}\right)$. So

$$
\begin{aligned}
\frac{9}{8} \mu_{-} C_{K} \varepsilon\left\|\left(\nabla \mathbf{u}_{1}^{\varepsilon}, \nabla \mathbf{u}_{2}^{\varepsilon}\right)\right\|_{0, \Omega_{1}^{\varepsilon} \times \Omega_{2}^{\varepsilon}}^{2} \leq & \frac{\left(h^{*}\right)^{2}}{\mu_{-} C_{K}}\left\|\left(\nabla \hat{\mathbf{f}}_{1}, \nabla \hat{\mathbf{f}}_{2}\right)\right\|_{0, \Omega_{1} \times \Omega_{2}}^{2} \\
& +\left(\frac{\left(\lambda_{+}\right)^{2}}{\mu_{-} C_{K}}+\frac{\mu_{+} C_{K}}{2}+\frac{4 \mu_{+}}{C_{K}}\right)\left\|\left(\nabla \hat{G}_{1}, \nabla \hat{G}_{2}\right)\right\|_{0, \Omega_{1} \times \Omega_{2}}^{2} .
\end{aligned}
$$

Thus

$$
\varepsilon\left\|\left(\nabla \mathbf{u}_{1}^{\varepsilon}, \nabla \mathbf{u}_{2}^{\varepsilon}\right)\right\|_{0, \Omega_{1}^{\varepsilon} \times \Omega_{2}^{\varepsilon}}^{2} \leq C,
$$

where,

$$
\begin{aligned}
\varepsilon\left\|\left(\nabla \mathbf{u}_{1}^{\varepsilon}, \nabla \mathbf{u}_{2}^{\varepsilon}\right)\right\|_{0, \Omega_{1}^{\varepsilon} \times \Omega_{2}^{\varepsilon}}^{2}= & \varepsilon^{2} \sum_{1 \leq i, j \leq 2}\left\|\left(\frac{\partial \hat{u}_{1 i}^{\varepsilon}}{\partial x_{j}}, \frac{\partial \hat{u}_{2 i}^{\varepsilon}}{\partial x_{j}}\right)\right\|_{0, \Omega_{1} \times \Omega_{2}}^{2}+\varepsilon^{2}\left\|\left(\frac{\partial \hat{u}_{13}^{\varepsilon}}{\partial z}, \frac{\partial \hat{u}_{23}^{\varepsilon}}{\partial z}\right)\right\|_{0, \Omega_{1} \times \Omega_{2}}^{2} \\
& +\sum_{1 \leq i \leq 2}\left(\left\|\left(\frac{\partial \hat{u}_{1 i}^{\varepsilon}}{\partial z}, \frac{\partial \hat{u}_{2 i}^{\varepsilon}}{\partial z}\right)\right\|_{0, \Omega_{1} \times \Omega_{2}}^{2}+\varepsilon^{4}\left\|\left(\frac{\partial \hat{u}_{13}^{\varepsilon}}{\partial x_{i}}, \frac{\partial \hat{u}_{23}^{\varepsilon}}{\partial x_{i}}\right)\right\|_{0, \Omega_{1} \times \Omega_{2}}^{2}\right) \leq C, \\
C= & \frac{8}{9 \mu-C_{K}} c_{0}, \\
c_{0}= & \frac{\left(h^{*}\right)^{2}}{\mu_{-} C_{K}}\left\|\left(\nabla \hat{\mathbf{f}}_{1}, \nabla \hat{\mathbf{f}}_{2}\right)\right\|_{0, \Omega_{1} \times \Omega_{2}}^{2} \\
& +\left(\frac{\left(\lambda_{+}\right)^{2}}{\mu_{-} C_{K}}+\frac{\mu_{+} C_{K}}{2}+\frac{4 \mu_{+}}{C_{K}}\right)\left\|\left(\nabla \hat{G}_{1}, \nabla \hat{G}_{2}\right)\right\|_{0, \Omega_{1} \times \Omega_{2}}^{2} .
\end{aligned}
$$

This completes the proof.

\section{Study of the limit problem}

Theorem 5.1. Under the same assumptions of Theorem 4.1, there exists $\left(\mathbf{u}_{1}^{\star}, \mathbf{u}_{2}^{\star}\right)=\left(u_{1 i}^{\star}, u_{2 i}^{\star}\right)$ in $H_{z}$, $i=1,2$, such that

$$
\begin{aligned}
& \left(\hat{u}_{1 i}^{\varepsilon}, \hat{u}_{2 i}^{\varepsilon}\right) \rightarrow\left(u_{1 i}^{\star}, u_{2 i}^{\star}\right),(1 \leq i \leq 2) \quad \text { weakly in } H_{z} \\
& \left(\varepsilon \frac{\partial \hat{u}_{1 i}^{\varepsilon}}{\partial x_{j}}, \varepsilon \frac{\partial \hat{u}_{2 i}^{\varepsilon}}{\partial x_{j}}\right) \rightarrow(0,0),(1 \leq i, j \leq 2) \text { weakly in } L^{2}\left(\Omega_{1}\right) \times L^{2}\left(\Omega_{2}\right) \\
& \left(\varepsilon^{2} \frac{\partial \hat{u}_{13}^{\varepsilon}}{\partial x_{i}}, \varepsilon^{2} \frac{\partial \hat{u}_{23}^{\varepsilon}}{\partial x_{i}}\right) \rightarrow(0,0),(1 \leq i \leq 2) \text { weakly in } L^{2}\left(\Omega_{1}\right) \times L^{2}\left(\Omega_{2}\right) \\
& \left(\varepsilon \frac{\partial \hat{u}_{13}^{\varepsilon}}{\partial z}, \varepsilon \frac{\partial \hat{u}_{23}^{\varepsilon}}{\partial z}\right) \rightarrow(0,0) \text { weakly in } L^{2}\left(\Omega_{1}\right) \times L^{2}\left(\Omega_{2}\right) \\
& \left(\varepsilon \hat{u}_{13}^{\varepsilon}, \varepsilon \hat{u}_{23}^{\varepsilon}\right) \rightarrow(0,0) \text { weakly in } L^{2}\left(\Omega_{1}\right) \times L^{2}\left(\Omega_{2}\right) .
\end{aligned}
$$

Proof. The convergences of (5.1) -5.5) are a direct result of inequalities 4.5).

Theorem 5.2. Under the same assumptions of Theorem 5.1, the solution $\left(\mathbf{u}_{1}^{\star}, \mathbf{u}_{2}^{\star}\right)$ satisfies

$$
\left(-\mu_{1} \frac{\partial^{2} \mathbf{u}_{1}^{\star}}{\partial z^{2}},-\mu_{2} \frac{\partial^{2} \mathbf{u}_{2}^{\star}}{\partial z^{2}}\right)=\left(\hat{\mathbf{f}}_{1}, \hat{\mathbf{f}}_{2}\right) \text { in } L^{2}\left(\Omega_{1}\right)^{2} \times L^{2}\left(\Omega_{2}\right)^{2},
$$




$$
\begin{aligned}
\mu_{1} & \sum_{1 \leq i \leq 2} \int_{\Omega_{1}} \frac{\partial u_{1 i}^{\star}}{\partial z} \cdot \frac{\partial}{\partial z}\left(\hat{\varphi}_{1 i}-u_{1 i}^{\star}\right) d x^{\prime} d z+\mu_{2} \sum_{1 \leq i \leq 2} \int_{\Omega_{2}} \frac{\partial u_{2 i}^{\star}}{\partial z} \cdot \frac{\partial}{\partial z}\left(\hat{\varphi}_{2 i}-u_{2 i}^{\star}\right) d x^{\prime} d z \\
& +\hat{J}\left(\hat{\varphi}_{1}, \hat{\varphi}_{2}\right)-\hat{J}\left(\mathbf{u}_{1}^{\star}, \mathbf{u}_{2}^{\star}\right) \\
& \geq \sum_{1 \leq i \leq 2} \int_{\Omega_{1}} \hat{f}_{1 i}\left(\hat{\varphi}_{1 i}-u_{1 i}^{\star}\right) d x^{\prime} d z+\sum_{1 \leq i \leq 2} \int_{\Omega_{2}} \hat{f}_{2 i}\left(\hat{\varphi}_{2 i}-u_{2 i}^{\star}\right) d x^{\prime} d z, \forall\left(\hat{\varphi}_{1}, \hat{\varphi}_{2}\right) \in \overline{V\left(\Omega_{1}\right)} \times \overline{V\left(\Omega_{2}\right)} .
\end{aligned}
$$

Proof. Using the convergence results of Theorem 5.1 in the variational inequality (4.4), and as $\hat{J}$ is convex and lower semi-continuous, we obtain

$$
\begin{aligned}
& \mu_{1} \sum_{1 \leq i \leq 2} \int_{\Omega_{1}} \frac{\partial \hat{u}_{1 i}^{\star}}{\partial z} \frac{\partial}{\partial z}\left(\hat{\varphi}_{1 i}-\hat{u}_{1 i}^{\star}\right) d x^{\prime} d z+\mu_{2} \sum_{1 \leq i \leq 2} \int_{\Omega_{2}} \frac{\partial \hat{u}_{2 i}^{\star}}{\partial z} \frac{\partial}{\partial z}\left(\hat{\varphi}_{2 i}-\hat{u}_{2 i}^{\star}\right) d x^{\prime} d z+\hat{J}\left(\hat{\varphi}_{1}, \hat{\varphi}_{2}\right)-\hat{J}\left(\mathbf{u}_{1}^{\star}, \mathbf{u}_{2}^{\star}\right) \\
& \quad \geq \sum_{1 \leq i \leq 2} \int_{\Omega_{1}} \hat{f}_{1 i}\left(\hat{\varphi}_{1 i}-u_{1 i}^{\star}\right) d x^{\prime} d z+\sum_{1 \leq i \leq 2} \int_{\Omega_{2}} \hat{f}_{2 i}\left(\hat{\varphi}_{2 i}-u_{2 i}^{\star}\right) d x^{\prime} d z \quad \forall\left(\hat{\varphi}_{1}, \hat{\varphi}_{2}\right) \in V .
\end{aligned}
$$

Now we choose $\hat{\varphi}_{1 i}=u_{1 i}^{\star} \pm \psi_{1 i}, \hat{\varphi}_{2 i}=u_{2 i}^{\star} \pm \psi_{2 i}, i=1,2$, with $\left(\psi_{1 i}, \psi_{2 i}\right)_{1 \leq i \leq 2} \in H_{0}^{1}\left(\Omega_{1}\right) \times H_{0}^{1}\left(\Omega_{2}\right)$, we find

$$
\begin{aligned}
\mu_{1} & \sum_{1 \leq i \leq 2} \int_{\Omega_{1}} \frac{\partial u_{1 i}^{\star}}{\partial z} \frac{\partial \psi_{1 i}}{\partial z} d x^{\prime} d z+\mu_{2} \sum_{1 \leq i \leq 2} \int_{\Omega_{2}} \frac{\partial u_{2 i}^{\star}}{\partial z} \frac{\partial \psi_{2 i}}{\partial z} d x^{\prime} d z \\
& =\sum_{1 \leq i \leq 2} \int_{\Omega_{1}} \hat{f}_{1 i} \psi_{1 i} d x^{\prime} d z+\sum_{1 \leq i \leq 2} \int_{\Omega_{2}} \hat{f}_{2 i} \psi_{2 i} d x^{\prime} d z
\end{aligned}
$$

Utilising Green's formula, we deduce

$$
\begin{aligned}
& -\int_{\Omega_{1}} \mu_{1} \frac{\partial}{\partial z}\left(\frac{\partial u_{1 i}^{\star}}{\partial z}\right) \psi_{1 i} d x^{\prime} d z-\int_{\Omega_{2}} \mu_{2} \frac{\partial}{\partial z}\left(\frac{\partial u_{2 i}^{\star}}{\partial z}\right) \psi_{2 i} d x^{\prime} d z \\
& =\int_{\Omega_{1}} \hat{f}_{1 i} \psi_{1 i} d x^{\prime} d z+\int_{\Omega_{2}} \hat{f}_{2 i} \psi_{2 i} d x^{\prime} d z .
\end{aligned}
$$

So,

$$
\left\langle\left(-\mu_{1} \frac{\partial^{2} u_{1 i}^{\star}}{\partial z^{2}}-\hat{f}_{1 i},-\mu_{2} \frac{\partial^{2} u_{2 i}^{\star}}{\partial z^{2}}-\hat{f}_{2 i}\right),\left(\psi_{1 i}, \psi_{2 i}\right)\right\rangle=0, i=1,2, \forall\left(\psi_{1 i}, \psi_{2 i}\right) \in H_{0}^{1}\left(\Omega_{1}\right) \times H_{0}^{1}\left(\Omega_{2}\right),
$$

thus

$$
\left(-\mu_{1} \frac{\partial^{2} u_{1 i}^{\star}}{\partial z^{2}},-\mu_{2} \frac{\partial^{2} u_{2 i}^{\star}}{\partial z^{2}}\right)=\left(\hat{f}_{1 i}, \hat{f}_{2 i}\right), i=1,2 \text { in } H^{-1}\left(\Omega_{1}\right) \times H^{-1}\left(\Omega_{2}\right)
$$

and as $\left(\hat{f}_{1 i}, \hat{f}_{2 i}\right) \in L^{2}\left(\Omega_{1}\right) \times L^{2}\left(\Omega_{2}\right)$, then 5.8 is valid in $L^{2}\left(\Omega_{1}\right) \times L^{2}\left(\Omega_{2}\right)$.

Theorem 5.3. Under the assumptions of preceding theorems, we have the following inequality

$$
\begin{gathered}
\mu_{1} \pi_{1}^{\star}=\mu_{2} \pi_{2}^{\star} \text { in } L^{2}(\omega)^{2}, \\
\int_{\omega} \widehat{k}\left(\left|\psi+s_{1}^{\star}-s_{2}^{\star}-s\right|-\left|s_{1}^{\star}-s_{2}^{\star}-s\right|\right) d x^{\prime}-\int_{\omega} \mu_{l} \pi_{l}^{\star} \psi d x^{\prime} \geq 0 \quad \forall \psi \in L^{2}(\omega)^{2},
\end{gathered}
$$




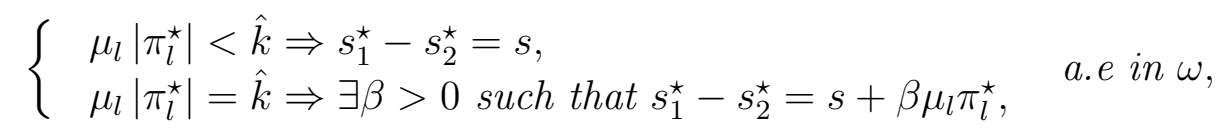

where

$$
s_{l}^{\star}=\mathbf{u}_{l}^{\star}\left(x^{\prime}, 0\right), \quad \pi_{l}^{\star}=\frac{\partial \mathbf{u}_{l}^{\star}}{\partial z}\left(x^{\prime}, 0\right), l=1,2 .
$$

Also $\pi_{l}^{\star}, s_{l}^{\star}$ satisfy the following:

$$
\int_{\omega}\left(\tilde{\mathcal{F}}+\mu_{1} \int_{0}^{h} \mathbf{u}_{1}^{\star}\left(x^{\prime}, y\right) d y+\mu_{2} \int_{-h}^{0} \mathbf{u}_{2}^{\star}\left(x^{\prime}, y\right) d y\right) . \nabla \psi\left(x^{\prime}\right) d x^{\prime}=0 \quad \forall \psi \in H^{1}(\omega),
$$

where

$$
\begin{aligned}
& \mathcal{F}_{1}\left(x^{\prime}, y\right)=\int_{0}^{y} \int_{0}^{\xi} \widehat{\mathbf{f}}_{1}\left(x^{\prime}, \theta\right) d \theta d \xi, \mathcal{F}_{2}\left(x^{\prime}, y\right)=\int_{y}^{0} \int_{\xi}^{0} \widehat{\mathbf{f}}_{2}\left(x^{\prime}, \theta\right) d \theta d \xi \\
& \mathcal{F}\left(x^{\prime}, y\right)=\mathcal{F}_{1}\left(x^{\prime}, y\right)+\mathcal{F}_{2}\left(x^{\prime},-y\right) \text { and } \widetilde{\mathcal{F}}\left(x^{\prime}\right)=\int_{0}^{h} \mathcal{F}\left(x^{\prime}, y\right) d y-h \mathcal{F}\left(x^{\prime}, h\right) .
\end{aligned}
$$

Proof. The variational inequality (4.4) becomes

$$
\begin{aligned}
\varepsilon^{2} \sum_{1 \leq i, j, l \leq 2}\left\{\mu_{l} \int_{\Omega_{1}}\left(\frac{\partial \hat{u}_{l i}^{\varepsilon}}{\partial x_{j}}+\frac{\partial \hat{u}_{l j}^{\varepsilon}}{\partial x_{i}}\right) \frac{\partial}{\partial x_{j}}\left(\hat{\varphi}_{l i}-\hat{u}_{l i}^{\varepsilon}\right) d x^{\prime} d z\right\} \\
\quad+\sum_{1 \leq i, l \leq 2}\left\{\mu_{l} \int\left(\frac{\partial \hat{u}_{l i}^{\varepsilon}}{\partial z}+\varepsilon^{2} \frac{\partial \hat{u}_{l 3}^{\varepsilon}}{\partial x_{i}}\right)\left[\frac{\partial}{\partial z}\left(\hat{\varphi}_{l i}-\hat{u}_{l i}^{\varepsilon}\right)+\varepsilon^{2} \frac{\partial}{\partial x_{i}}\left(\hat{\varphi}_{l 3}-\hat{u}_{l 3}^{\varepsilon}\right)\right] d x^{\prime} d z\right\} \\
\quad+\varepsilon^{2} \sum_{1 \leq l \leq 2}\left(2 \mu_{l} \int_{\Omega_{1}} \frac{\partial \hat{u}_{l 3}^{\varepsilon}}{\partial z} \frac{\partial}{\partial z}\left(\hat{\varphi}_{l 3}-\hat{u}_{l 3}^{\varepsilon}\right) d x^{\prime} d z\right)+\varepsilon^{2} \sum_{1 \leq l \leq 2}\left(\lambda_{l} \int_{\Omega_{1}} d i v\left(\hat{u}_{l}^{\varepsilon}\right) d i v\left(\hat{\varphi}_{l}-\hat{u}_{l}^{\varepsilon}\right) d x^{\prime} d z\right) \\
\quad+\int_{\omega} \hat{k}\left|\left(\hat{\varphi}_{1 \tau}-\hat{\varphi}_{2 \tau}-s\right)\right| d x^{\prime}-\int \hat{k}_{\omega}\left|\left(\hat{\mathbf{u}}_{1 \tau}^{\varepsilon}-\hat{\mathbf{u}}_{2 \tau}^{\varepsilon}-s\right)\right| d x^{\prime} \\
\geq \sum_{1 \leq i \leq 2}\left(\int_{\Omega_{1}} \hat{f}_{1 i}\left(\hat{\varphi}_{1 i}-\hat{u}_{1 i}^{\varepsilon}\right) d x^{\prime} d z\right)+\varepsilon \int_{\Omega_{1}} \hat{f}_{13}\left(\hat{\varphi}_{13}-\hat{u}_{13}^{\varepsilon}\right) d x^{\prime} d z \\
\quad+\sum_{1 \leq i \leq 2}\left(\int_{\Omega_{2}} \hat{f}_{2 i}\left(\hat{\varphi}_{2 i}-\hat{u}_{1 i}^{\varepsilon}\right) d x^{\prime} d z\right)+\varepsilon \int_{\Omega_{2}} \hat{f}_{23}\left(\hat{\varphi}_{23}-\hat{u}_{23}^{\varepsilon}\right) d x^{\prime} d z .
\end{aligned}
$$

From [8, Lemma 5.3], we can choose $\hat{\varphi}_{1 i}=u_{1 i}^{\star}+\psi_{1 i}, \hat{\varphi}_{2 i}=u_{2 i}^{\star}+\psi_{2 i}, i=1,2$, with $\left(\psi_{1 i}, \psi_{2 i}\right) \in$ $H_{\Gamma_{1} \cup \Gamma_{L_{1}}}^{1}\left(\Omega_{1}\right) \times H_{\Gamma_{2} \cup \Gamma_{L_{2}}}^{1}\left(\Omega_{2}\right)$, where

$$
H_{\Gamma_{l} \cup \Gamma_{L_{l}}}^{1}\left(\Omega_{l}\right)=\left\{\varphi_{l} \in H^{1}\left(\Omega_{l}\right): \varphi_{l}=0 \text { on } \Gamma_{l} \cup \Gamma_{L_{l}}\right\}, l=1,2,
$$

then

$$
\mu_{1} \sum_{1 \leq i \leq 2} \int_{\Omega_{1}} \frac{\partial u_{1 i}^{\star}}{\partial z} \frac{\partial \psi_{1 i}}{\partial z} d x^{\prime} d z+\mu_{2} \sum_{1 \leq i \leq 2} \int_{\Omega_{2}} \frac{\partial u_{2 i}^{\star}}{\partial z} \frac{\partial \psi_{2 i}}{\partial z} d x^{\prime} d z+\hat{J}\left(\psi_{1}+s_{1}^{\star}, \psi_{2}+s_{2}^{\star}\right)-\hat{J}\left(s_{1}^{\star}, s_{2}^{\star}\right)
$$




$$
\geq \sum_{1 \leq i \leq 2} \int_{\Omega_{1}} \hat{f}_{1 i} \cdot \psi_{1 i} d x^{\prime} d z+\sum_{1 \leq i \leq 2} \int_{\Omega_{2}} \hat{f}_{2 i} . \psi_{2 i} d x^{\prime} d z
$$

Using Green's formula on each subdomain $\Omega_{l}^{\varepsilon}, l=1,2$, we get

$$
\begin{aligned}
& \sum_{1 \leq i \leq 2} \int_{\Omega_{1}}\left\{-\mu_{1} \frac{\partial^{2} u_{1 i}^{\star}}{\partial z^{2}}\right\} \psi_{1 i} d x^{\prime} d z+\int_{\omega} \mu_{1} \tau_{1}^{\star} \cdot \psi_{1} d \sigma+\sum_{1 \leq i \leq 2} \int_{\Omega_{2}}\left\{-\mu_{2} \frac{\partial^{2} u_{2 i}^{\star}}{\partial z^{2}}\right\} \psi_{2 i} d x^{\prime} d z \\
& \quad-\int_{\omega} \mu_{2} \tau_{2}^{\star} \cdot \psi_{2} d \sigma+\int_{\omega} \hat{k}\left(\left|\psi_{1}-\psi_{2}+s_{1}^{\star}-s_{2}^{\star}-s\right|-\left|s_{1}^{\star}-s_{2}^{\star}-s\right|\right) d x^{\prime} \\
& \geq \sum_{1 \leq i \leq 2} \int_{\Omega_{1}} \hat{f}_{1 i} \psi_{1 i} d x^{\prime} d z+\sum_{1 \leq i \leq 2} \int_{\Omega_{2}} \hat{f}_{2 i} \psi_{2 i} d x^{\prime} d z .
\end{aligned}
$$

By (5.4), we deduce for $\psi_{l} \in H_{\Gamma_{l} \cup \Gamma_{L_{l}}}^{1}\left(\Omega_{l}\right)^{2}, l=1,2$,

$$
\int_{\omega} \hat{k}\left(\left|\psi_{1}-\psi_{2}+s_{1}^{\star}-s_{2}^{\star}-s\right|-\left|s_{1}^{\star}-s_{2}^{\star}-s\right|\right) d x^{\prime}-\int_{\omega}\left(\mu_{1} \pi_{1}^{\star} \psi_{1}-\mu_{2} \pi_{2}^{\star} \psi_{2}\right) d x^{\prime} \geq 0 .
$$

This inequality holds for all $\psi_{l} \in \mathcal{D}(\omega)^{2}, l=1,2$, and by the density of $\mathcal{D}(\omega)$ in $L(\omega)$ we deduce

$$
\begin{aligned}
\int_{\omega} \hat{k} & \left(\left|\psi_{1}-\psi_{2}+s_{1}^{\star}-s_{2}^{\star}-s\right|-\left|s_{1}^{\star}-s_{2}^{\star}-s\right|\right) d x^{\prime} \\
& -\int_{\omega}\left(\mu_{1} \pi_{1}^{\star} \psi_{1}-\mu_{2} \pi_{2}^{\star} \cdot \psi_{2}\right) d x^{\prime} \geq 0 \quad \forall \psi_{1}, \psi_{2} \in L^{2}(\omega)^{2} .
\end{aligned}
$$

In the particular case for $\psi_{1}=\psi_{2}= \pm \psi$, we find

$$
\int_{\omega}\left(\mu_{1} \pi_{1}^{\star}-\mu_{2} \pi_{2}^{\star}\right) \cdot \psi d x^{\prime}=0 \forall \psi \in L^{2}(\omega)^{2},
$$

which implies (5.9).

From (5.9) and (5.13), we deduce directly the inequality (5.10). The proof of (5.11) are similar to those given in case of the problem of fluids (see. [4]).

To prove (5.12), we integrate twice the first equation of $(5.6)$ between 0 and $z$, and the second between $z$ and 0 , we obtain

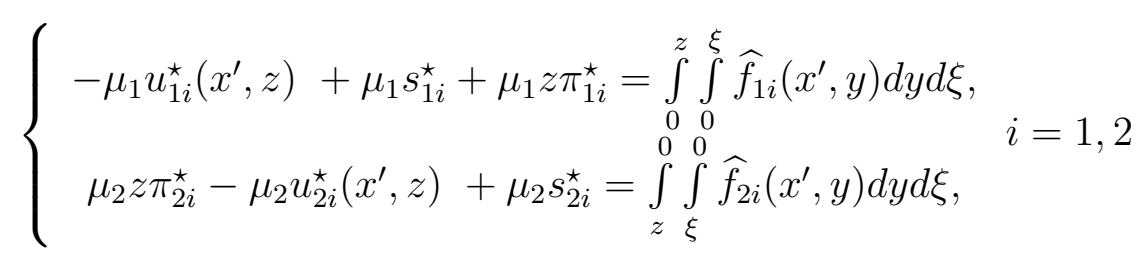

then for $z=h$ in the first equation of (5.14) and in the second $z=-h$, we find

$$
\left\{\begin{aligned}
\mu_{1} s_{1 i}^{\star}+\mu_{1} h \pi_{1 i}^{\star} & =\int_{0}^{h} \int_{0}^{\xi} \widehat{f}_{1 i}\left(x^{\prime}, y\right) d y d \xi, \\
-\mu_{2} h \pi_{2 i}^{\star}+\mu_{2} s_{2 i}^{\star} & =\int_{-h}^{0} \int_{\xi}^{0} \widehat{f}_{2 i}\left(x^{\prime}, y\right) d y d \xi .
\end{aligned}\right.
$$


By (5.9), we deduce

$$
\mu_{1} s_{1}^{\star}+\mu_{2} s_{2}^{\star}=\int_{0}^{h} \int_{0}^{\xi} \widehat{f}_{1 i}\left(x^{\prime}, y\right) d y d \xi+\int_{-h}^{0} \int_{\xi}^{0} \widehat{f}_{2 i}\left(x^{\prime}, y\right) d y d \xi
$$

Now, we integrate the first equation of (5.14) between 0 and $h$, and the second between $-h$ and 0 , we obtain

$$
\left\{\begin{aligned}
-\mu_{1} \int_{0}^{h} \mathbf{u}_{1}^{\star}\left(x^{\prime}, y\right) d y+\mu_{1} s_{1}^{\star} h+\frac{h^{2}}{2} \mu_{1} \pi_{1}^{\star}=\int_{0}^{h} \mathcal{F}_{1}\left(x^{\prime}, y\right) d y \\
-\frac{h^{2}}{2} \mu_{2} \pi_{2}^{\star}-\mu_{2} \int_{-h}^{0} \mathbf{u}_{2}^{\star}\left(x^{\prime}, y\right) d y+\mu_{2} s_{2}^{\star} h=\int_{-h}^{0} \mathcal{F}_{2}\left(x^{\prime}, y\right) d y
\end{aligned}\right.
$$

with

$$
\mathcal{F}_{1}\left(x^{\prime}, y\right)=\int_{0}^{y} \int_{0}^{\xi} \widehat{\mathbf{f}_{1}}\left(x^{\prime}, \theta\right) d \theta d \xi \text { et } \mathcal{F}_{2}\left(x^{\prime}, y\right)=\int_{y}^{0} \int_{\xi}^{0} \widehat{\mathbf{f}_{2}}\left(x^{\prime}, \theta\right) d \theta d \xi
$$

Therefore,

$$
h\left(\mu_{1} s_{1}^{\star}+\mu_{2} s_{2}^{\star}\right)-\mu_{1} \int_{0}^{h} \mathbf{u}_{1}^{\star}\left(x^{\prime}, y\right) d y-\mu_{2} \int_{-h}^{0} \mathbf{u}_{2}^{\star}\left(x^{\prime}, y\right) d y=\int_{0}^{h} \mathcal{F}\left(x^{\prime}, y\right) d y
$$

with

$$
\mathcal{F}\left(x^{\prime}, y\right)=\mathcal{F}_{1}\left(x^{\prime}, y\right)+\mathcal{F}_{2}\left(x^{\prime},-y\right)
$$

which gives 5.12 .

Theorem 5.4. Under the assumptions of Theorem 5.1, the solution $\left(\mathbf{u}_{1}^{\star}, \mathbf{u}_{2}^{\star}\right)$ of the limit problem (5.6) -(5.7) is unique in $H_{z}$.

Proof. Let $\left(\mathbf{u}_{1}^{\star}, \mathbf{u}_{2}^{\star}\right)$ and $\left(\mathbf{v}_{1}^{\star}, \mathbf{v}_{2}^{\star}\right)$ be the two solutions of the limit problem (5.4)-(5.5), then

$$
\begin{aligned}
& \mu_{1} \sum_{i=1}^{2} \int_{\Omega_{1}} \frac{\partial u_{1 i}^{\star}}{\partial z} \frac{\partial}{\partial z}\left(\hat{\varphi}_{1 i}-u_{1 i}^{\star}\right) d x^{\prime} d z+\mu_{2} \sum_{i=1}^{2} \int_{\Omega_{2}} \frac{\partial u_{2 i}^{\star}}{\partial z} \frac{\partial}{\partial z}\left(\hat{\varphi}_{2 i}-u_{2 i}^{\star}\right) d x^{\prime} d z \\
& \quad+J\left(\hat{\varphi}_{1}, \hat{\varphi}_{2}\right)-J\left(\mathbf{u}_{1}^{\star}, \mathbf{u}_{2}^{\star}\right) \\
& \quad \geq \sum_{i=1}^{2} \int_{\Omega_{1}} \hat{f}_{1 i}\left(\hat{\varphi}_{1 i}-u_{1 i}^{\star}\right) d x^{\prime} d z+\sum_{i=1}^{2} \int_{\Omega_{2}} \hat{f}_{2 i}\left(\hat{\varphi}_{2 i}-u_{2 i}^{\star}\right) d x^{\prime} d z, \forall\left(\hat{\varphi}_{1}, \hat{\varphi}_{2}\right) \in \overline{V\left(\Omega_{1}\right)} \times \overline{V\left(\Omega_{2}\right)}, \\
& \mu_{1} \sum_{i=1}^{2} \int_{\Omega_{1}} \frac{\partial v_{1 i}^{\star}}{\partial z} \frac{\partial}{\partial z}\left(\hat{\varphi}_{1 i}-v_{1 i}^{\star}\right) d x^{\prime} d z+\mu_{2} \sum_{i=1}^{2} \int_{\Omega_{2}} \frac{\partial v_{2 i}^{\star}}{\partial z} \frac{\partial}{\partial z}\left(\hat{\varphi}_{2 i}-v_{2 i}^{\star}\right) d x^{\prime} d z \\
& \quad+J\left(\hat{\varphi}_{1}, \hat{\varphi}_{2}\right)-J\left(\mathbf{v}_{1}^{\star}, \mathbf{v}_{2}^{\star}\right) \\
& \quad \geq \sum_{i=1}^{2} \int_{\Omega_{1}} \hat{f}_{1 i}\left(\hat{\varphi}_{1 i}-v_{1 i}^{\star}\right) d x^{\prime} d z+\sum_{i=1}^{2} \int_{\Omega_{2}} \hat{f}_{2 i}\left(\hat{\varphi}_{2 i}-v_{2 i}^{\star}\right) d x^{\prime} d z, \forall\left(\hat{\varphi}_{1}, \hat{\varphi}_{2}\right) \in \overline{V\left(\Omega_{1}\right)} \times \overline{V\left(\Omega_{2}\right)} .
\end{aligned}
$$


Taking $\widehat{\varphi}_{1}=\mathbf{v}_{1}^{\star}$ and $\widehat{\varphi}_{2}=\mathbf{v}_{2}^{\star}$ in (5.17), then $\widehat{\varphi}_{1}=\mathbf{u}_{1}^{\star}$ and $\widehat{\varphi}_{2}=\mathbf{u}_{2}^{\star}$ in 5.18 and summing the two inequalities, we find for $\bar{W}_{1}=\mathbf{u}_{1}^{\star}-\mathbf{v}_{1}^{\star}$ and $\bar{W}_{2}=\mathbf{u}_{2}^{\star}-\mathbf{v}_{2}^{\star}$,

$$
\mu_{1} \sum_{i=1}^{2} \int_{\Omega_{1}} \frac{\partial}{\partial z}\left(\bar{W}_{1 i}\right) \frac{\partial}{\partial z}\left(\bar{W}_{1 i}\right) d x^{\prime} d z+\mu_{2} \sum_{i=1}^{2} \int_{\Omega_{2}} \frac{\partial}{\partial z}\left(\bar{W}_{2 i}\right) \frac{\partial}{\partial z}\left(\bar{W}_{2 i}\right) d x^{\prime} d z \leq 0
$$

which implies

SO

$$
\mu_{1}\left\|\frac{\partial}{\partial z}\left(\overline{W_{1}}\right)\right\|_{0, \Omega_{1}}^{2}+\mu_{2}\left\|\frac{\partial}{\partial z}\left(\overline{W_{2}}\right)\right\|_{0, \Omega_{2}}^{2} \leq 0
$$

$$
\left\|\frac{\partial}{\partial z} \bar{W}_{1}\right\|_{0, \Omega_{1}}^{2}=0 \text { and }\left\|\frac{\partial}{\partial z} \bar{W}_{2}\right\|_{0, \Omega_{2}}^{2}=0 .
$$

By Poincaré inequality, we deduce

$$
\left\|\bar{W}_{1}\right\|_{H_{z}\left(\Omega_{1}\right)}=0 \text { and }\left\|\bar{W}_{2}\right\|_{H_{z}\left(\Omega_{2}\right)}=0 .
$$

So $\left\|\left(\bar{W}_{1}, \bar{W}_{2}\right)\right\|_{H_{z}}=0$.

We deduce that $\left(\mathbf{u}_{1}^{\star}, \mathbf{u}_{2}^{\star}\right)=\left(\mathbf{v}_{1}^{\star}, \mathbf{v}_{2}^{\star}\right)$ almost everywhere in $H_{z}$.

\section{References}

[1] A. Atangana, A novel model for the Lassa Hemorrhagic Fever: Deathly Disease for Pregnant Women, Neural Comput. Appl., 26 (2015), 1895-1903. 1]

[2] A. Atangana, E. F. Doungmo Goufo, A model of the groundwater flowing within a leaky aquifer using the concept of local variable order derivative, J. Nonlinear Sci. Appl., 8 (2015), 763-775. 1

[3] H. M. Baskonus, H. Bulut, New hyperbolic function solutions for some nonlinear partial differential equation arising in mathematical physics, Entropy, 17 (2015), 4255-4270. 1

[4] G. Bayada, M. Boukrouche, On a free boundary problem for the Reynolds equation derived from the Stokes systems with Tresca boundary conditions, J. Math. Anal. Appl., 282 (2003), 212-231. 2, 5

[5] G. Bayada, K. Lhalouani, Asymptotic and numerical analysis for unilateral contact problem with Coulomb's friction between an elastic body and a thin elastic soft layer, Asymptot. Anal., 25 (2001), 329-362. 1

[6] H. Benseridi, M. Dilmi, Some inequalities and asymptotic behavior of a dynamic problem of linear elasticity, Georgian Math. J., 20 (2013), 25-41. 1

[7] M. Boukrouche, R. El mir, On a non-isothermal, non-Newtonian lubrication problem with Tresca law: Existence and the behavior of weak solutions, Nonlinear Anal., 9 (2008), 674-692. 1

[8] M. Boukrouche, G. Łukaszewicz, On a lubrication problem with Fourier and Tresca boundary conditions, Math. Models Methods Appl. Sci., 14 (2004), 913-941. 4.5

[9] M. Boukrouche, F. Saidi, Non-isothermal lubrication problem with Tresca fluid-solid interface law. Part I, Nonlinear Anal., 7 (2006), 1145-1166.

[10] M. Dilmi, H. Benseridi, A. Saadallah, Asymptotic analysis of a Bingham fluid in a thin domain with Fourier and Tresca boundary conditions, Adv. Appl. Math. Mech., 6 (2014), 797-810. 1

[11] G. Duvaut, J. L. Lions, Les Inéquations en Mécanique et en Physique, Dunod, Paris, (1972). 3.1

[12] N. Hemici, A. Matei, A frictionless contact problem with adhesion between two elastic bodies, An. Univ. Craiova Ser. Mat. Inform., 30 (2003), 90-99. 1 .

[13] J. Koko, Uzawa block relaxation domain decomposition method for the two-body contact problem with Tresca friction, Comput. Methods Appl. Mech. Engrg., 198 (2008), 420-431. 1

[14] X. Li, Symmetric Coupling of the Meshless Galerkin boundary node and finite element methods for Elasticity, CMES Comput. Model. Eng. Sci., 97 (2014), 483-507. 1

[15] X. Li, H. Chen, Y. Wang, Error analysis in Sobolev spaces for the improved moving least-square approximation and the improved element-free Galerkin method, Appl. Math. Comput., 262 (2015), 56-78. 1

[16] A. Saadallah, H. Benseridi, M. Dilmi, S. Drabla, Estimates for the asymptotic convergence of a nonisothermal linear elasticity with friction, Georgian Math. J., 23 (2016), to appear. 1 